\title{
Beam Steering of Wearable Antenna with Metamaterial Structures for Patient Monitoring
}

\author{
Parisa Shirvani, Farzad Khajeh-Khalili and Raed A. Abd-Alhameed \\ \{ shirvaniiiparisaaa@gmail.com ${ }^{1}$, khalili.farzad@gmail.com ${ }^{2}$, r.a.a.abd@bradford.ac.uk ${ }^{3}$ \} \\ Electrical and Biomedical Department, Sepahan Institute of Science and Technology of Higher \\ Education, Isfahan, Iran ${ }^{1}$ \\ Electrical Department, Kian Institute of Higher Education, Shahin Shahr, Isfahan, Iran ${ }^{2}$ \\ Bradford-Renduchintala Centre for Space AI, Faculty of Engineering and Informatics, University of \\ Bradford, Bradford BD7 1DP, $\mathrm{UK}^{3}$
}

\begin{abstract}
At $5.2 \mathrm{GHz}$, in the industrial, scientific, and medical (ISM) sub-channels, a unique metamaterial (MTM) structure is used to tilt the beam and boost the directivity of a wearable antenna. The antenna substrate in this design is made of a typical jean cloth, which is a wearable and flexible material. The dielectric constant of normal denim fabric is 2.17 , the thickness is $3.175 \mathrm{~mm}$, and the $\tan (\delta)$ value is 0.002 . The antenna's radiation beam is steered in the right direction for healthcare purposes utilising this architecture. The radiation beam is slanted by $9^{\circ}$ in the E-plane, according to the simulation results. At 5.2 $\mathrm{GHz}$, the gain was found to be $6.35 \mathrm{~dB}$. Additionally, the specific absorption rate (SAR) is ideal for medicinal applications. The results show that the proposed antenna is a viable biomedical technology option.
\end{abstract}

Keywords: Wearable; Metamaterial; Patient monitoring; Beam tilting.

\section{Introduction}

Recently, there has been an increase in the development of wearable smart systems that are utilised for patient monitoring, tracking, physical training, and rescue system geo-positioning. It is obvious that the constant development of wearable systems features radically improves the performance of telecommunication systems in the health care area. One of the vital components of medical/telecommunication systems is wearable antennas which faced many challenges such as low profile, high gain, and high sensitivity. In addition to telecommunication discussions about antennas, wearable antennas that are used as part of clothing should meet medical standards such as high flexibility, make the patient comfortable, and be resistant to patient movements. To achieve these aims, some researchers have focused on choosing the best material as a substrate of the wearable antenna [1,2]. Some characteristics such as low-cost, high flexibility, widely available, and small loss tangent are assessed to choose the best material for the substrate of wearable antenna. Reducing the specific absorption rate (SAR) by the body is another arguable issue about the wearable antenna. The SAR parameter indicates the rate of absorbed energy per unit mass by the human body when it is exposed to electromagnetic waves. The SAR value is defined by The United States (US) and European Union (EU) regulatory authorities to maintain safety restrictions [3]. Different methods to gain enhancement and bandwidth improvement of a wearable antenna are presented in $[4,5]$. Reconfiguring the 
radiation pattern is a key factor to enhance the performance of the antenna which leads to interference reduction and system security increment. Some artificial periodic structures such as metamaterial (MTM) [6,7], artificial magnetic conductor (AMC) [8], electromagnetic bandgap (EBG) [9], and frequency selective surface (FSS) [10] had been employed for providing the beam tilting. Metamaterial structures are artificial compounds with remarkable electric and magnetic properties which employed to a revolution in the performance of antennas such as gain enhancement, radiation patterns improvement and widening bandwidth, the smaller size of the antenna, beam tilting, etc [11,12]. In [6], a metamaterial structure with four switches is used to tilting the radiation beam at $28 \mathrm{GHz}$. This structure can tilt the radiation beam at angles in the E-plane. In addition, the gain enhancement is $24.8 \%$ and $22.4 \%$ for \pm tilting angles, respectively. In [13], a stairway-shaped resonator (SSR) as a novel metamaterial for beam tilting at $28 \mathrm{GHz}$ has been designed. Two-pin diodes are used in the gaps of the structure to create four states with different refractive indexes. In [14], a negative refractive index metamaterial structure is designed to beam tilting of a double-feed dielectric resonator antenna. The main beam of the antenna can be tilted from over the frequency range of 5-5.5 GHz.

The body of this paper is as follows: Section 2 introduces the recommended design and its performances. In Section 3, the simulation results of the proposed antenna characteristics will be accessible. Then the significant advantages of the proposed wearable antenna will be expressed. Finally, in Section 4, a conclusion will be presented.

\section{Proposed Design}

In this section, the model features of a wearable antenna with the metamaterial unit-cells for use in medical/telecommunication systems are accessible. A novel MTM structure is employed to beam tilt optimization of a wearable antenna in health care networks. The aim is to control the inclination of the tilt angle of the antenna to optimize a set of network performance indicators such as reducing unwanted interfaces and increasing healthcare network security. As a result, the health care network works better, and more people trust telemedicine applications.

\subsection{Antenna Design}

A modified wearable antenna at $5.2 \mathrm{GHz}$ is shown in Fig 1. The suggested antenna is a very thin conductive material connected to a feedline as an upper layer at which the signal is to be transmitted. The wearable and flexible standard denim fabric is utilized as a middle layer with dielectric constant $\varepsilon_{r}=2.17, \tan \delta=0.002$, and thickness $3.175 \mathrm{~mm}$. The most significant feature of this dielectric is its high flexibility, low cost, and availability. The ground plane is also the last thin conductive layer. Table 1 introduces the antenna structure elements. The simulations were done with CST software. 


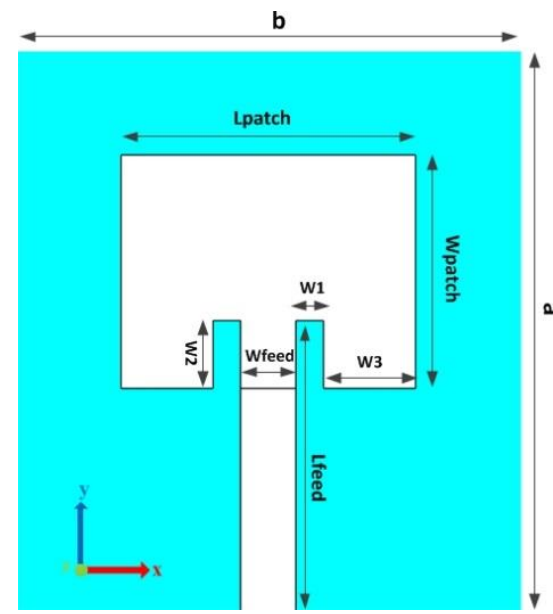

Table 1. The dimension of the patch antenna

Fig. 1. Configuration of the proposed wearable antenna

In Fig $2 \mathrm{a}$, the reflection at the input port is informed. According to this figure and assuming $\mathrm{S}_{11}$ $<-10 \mathrm{~dB}$, the frequency range of 5.16-5.4 GHz is covered. In Fig. 2 (b), the reported surface current at frequency $5.2 \mathrm{GHz}$ indicates good radiation.

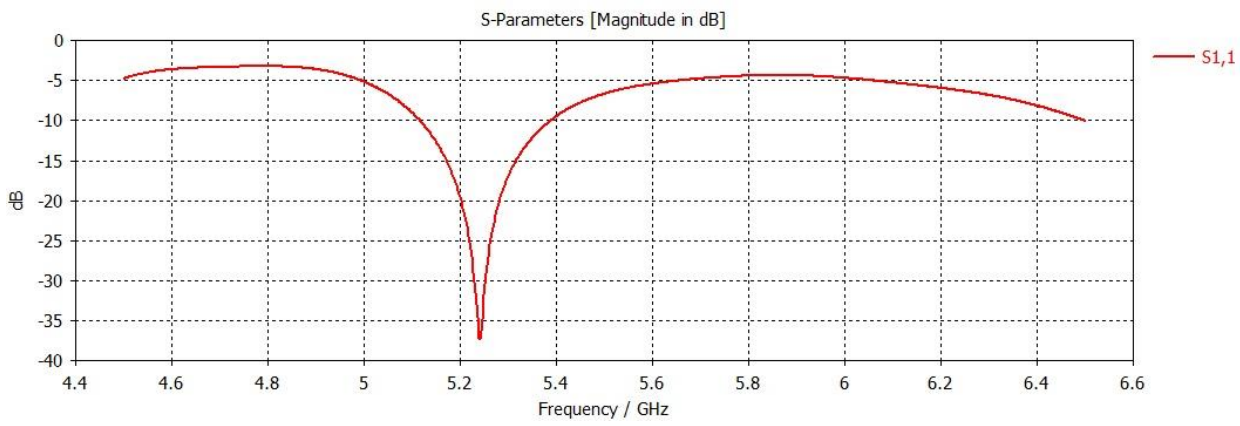

(a) 


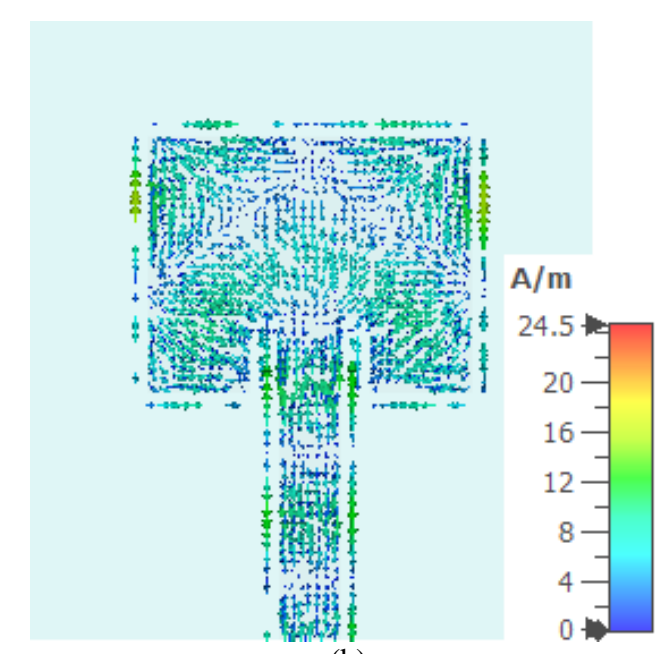

(b)

Fig. 2. The antenna results, (a) $S_{11}$, (b) surface current distribution.

\subsection{Metamaterial Design}

The geometric of the MTM cell is illustrated in Fig. 3, where a square ring with some crossdiagonal bars are notified. For full-wave analysis, along the $z$-axis, two waveguide ports are located, and in the $X Y$ and $Y Z$ planes, perfect magnetic and electric conductors (PMC and PEC) boundaries are located respectively. The dimensions of the proposed MTM unit cell are stated in Table (2).

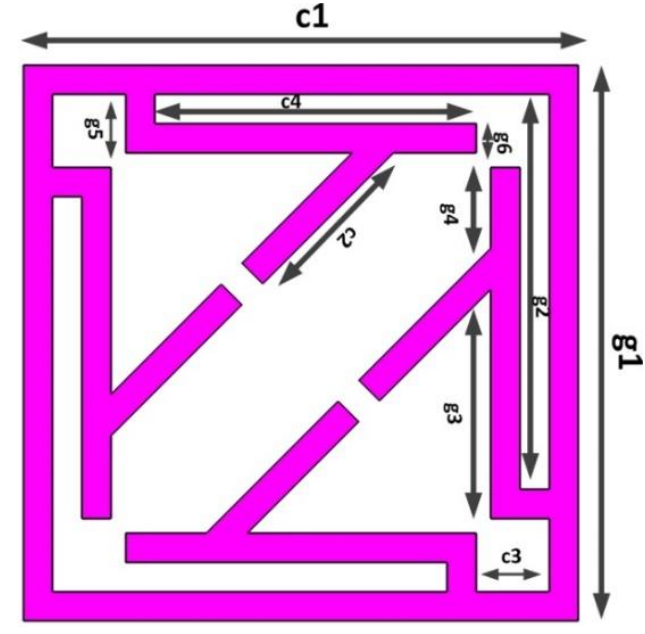

(a)

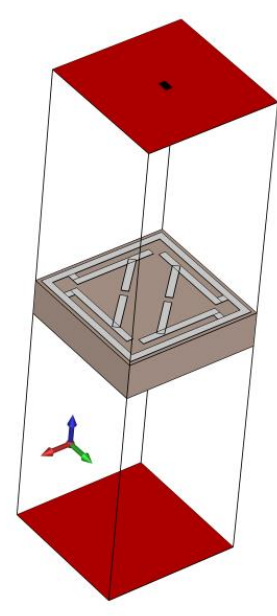

(b)

Fig. 3. The proposed metamaterial cell; (a) front view, (b) side view. 
Table 2. The dimension of MTM cell

\begin{tabular}{|c|c|c|c|c|c|c|c|c|c|c|}
\hline Parameters & $\mathrm{c} 1$ & $\mathrm{c} 2$ & $\mathrm{c} 3$ & $\mathrm{c} 4$ & $\mathrm{~g} 1$ & $\mathrm{~g} 2$ & $\mathrm{~g} 3$ & $\mathrm{~g} 4$ & $\mathrm{~g} 5$ & $\mathrm{~g} 6$ \\
\hline Dimensions (mm) & 5.7 & 1.9 & 0.75 & 3.3 & 5.7 & 4.05 & 2.35 & 0.83 & 0.6 & 0.3 \\
\hline
\end{tabular}

The S21 parameter and permeability of the recommended MTM structure are revealed in Fig 4. It displays the results of the $S_{21}$ from scattering parameters (S-parameters) as well as the permeability coefficient. According to Fig $3 \mathrm{a}$. and assuming $\mathrm{S}_{21}<-10 \mathrm{~dB}$, the bandwidth of this stopband is about $1 \mathrm{GHz}(5-6 \mathrm{GHz})$. Also, according to Fig 3b., within 3.2 to $5.7 \mathrm{GHz}$, the permeability is less than zero. The negativity of the permeability attests that this structure is a metamaterial unit-cell that can have a dramatic effect on improving the wearable antenna.

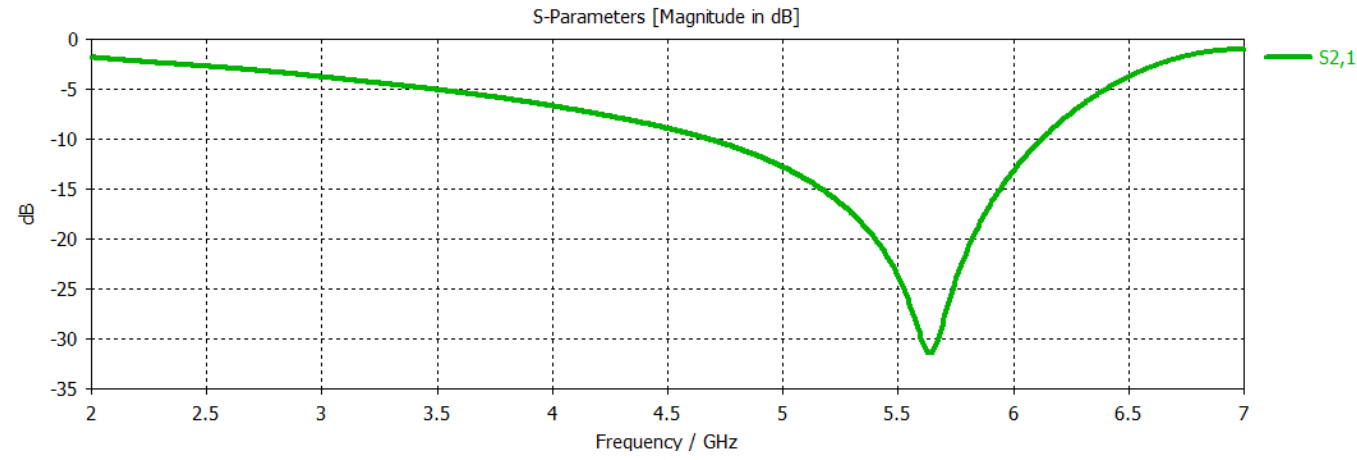

(a)

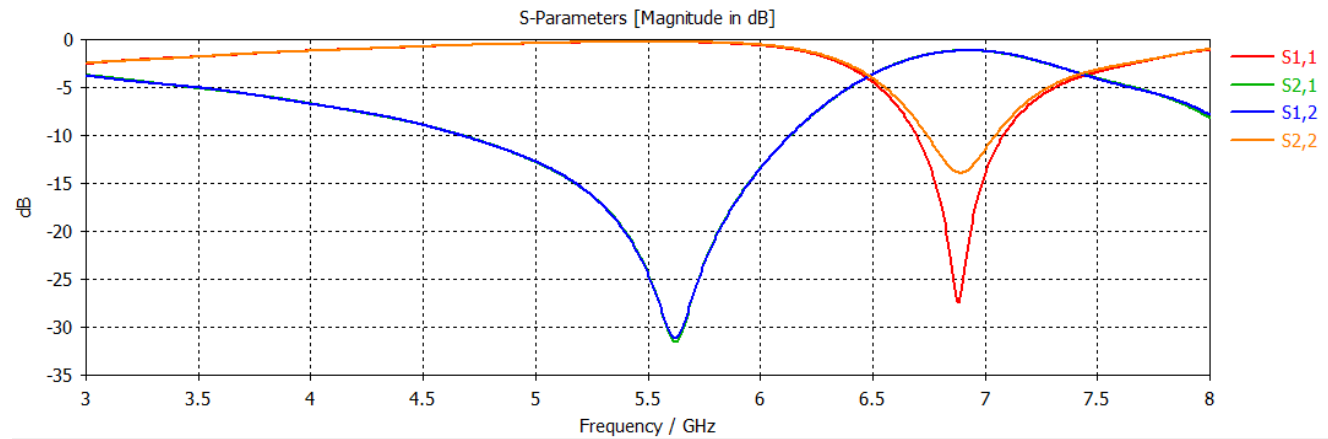

(b) 


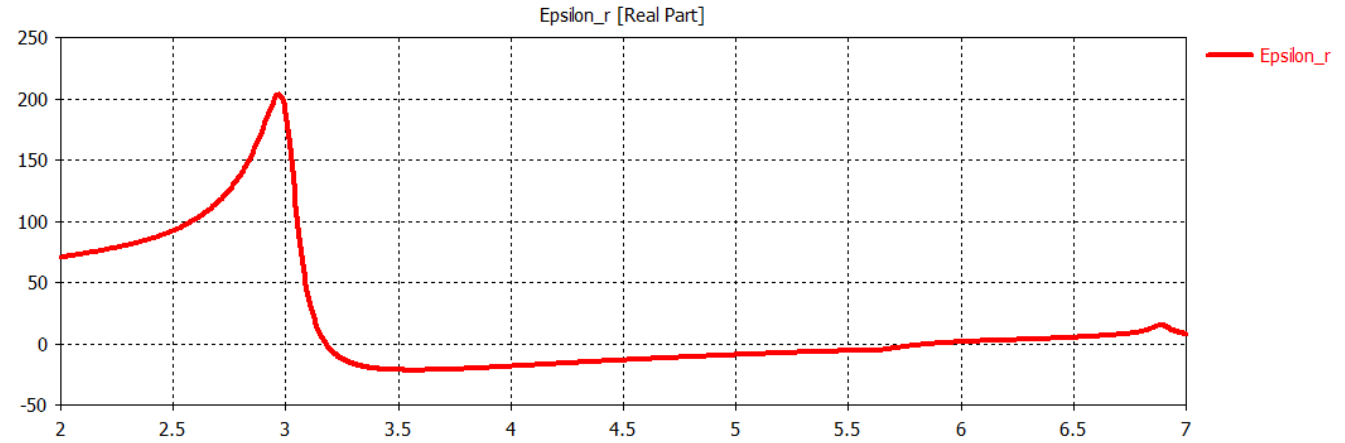

(c)

Fig. 4. (a) S21 parameter, (b) S-pars, (c) permeability of the present metamaterial cell.

To better justify the proposed MTM unit-cell behaviour, a report on the changes at the dimensions and the expression of its result in the performance of the structure is presented. The slot size effect on the $S_{21}$ parameter is shown in Fig 5. According to this figure, with increasing gap size, the transmission-zero has changed to lower frequency values. Note that this feature proves the "tunability" of the proposed structure. In [15], this issue has been thoroughly investigated in the same way.

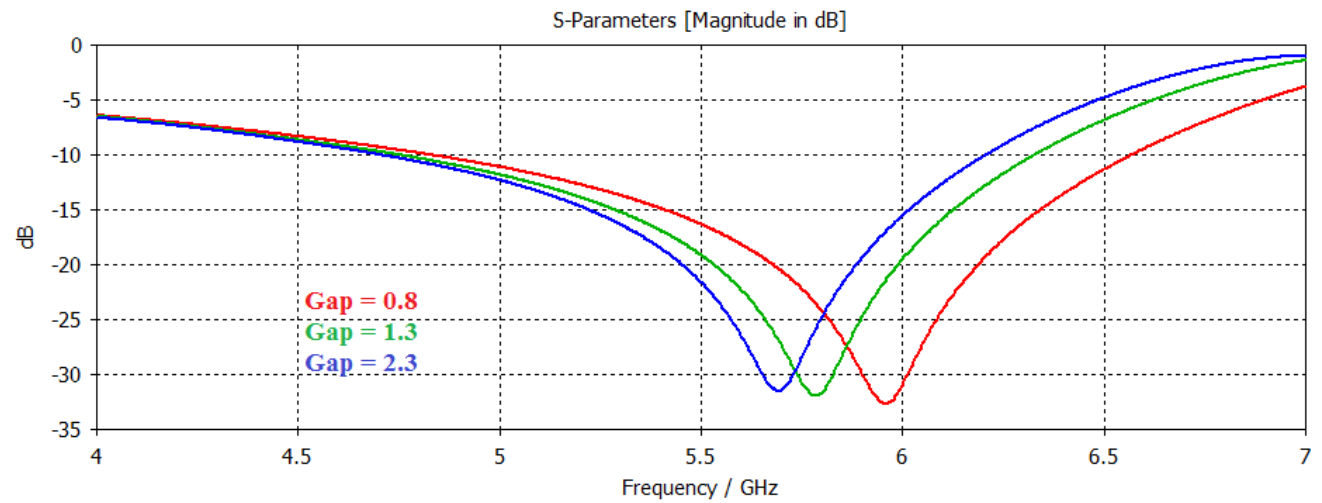

Fig. 5: $S_{21}$ parameter with different gap sizes

The slot size effect on the permeability parameter is shown in Figure 6. 


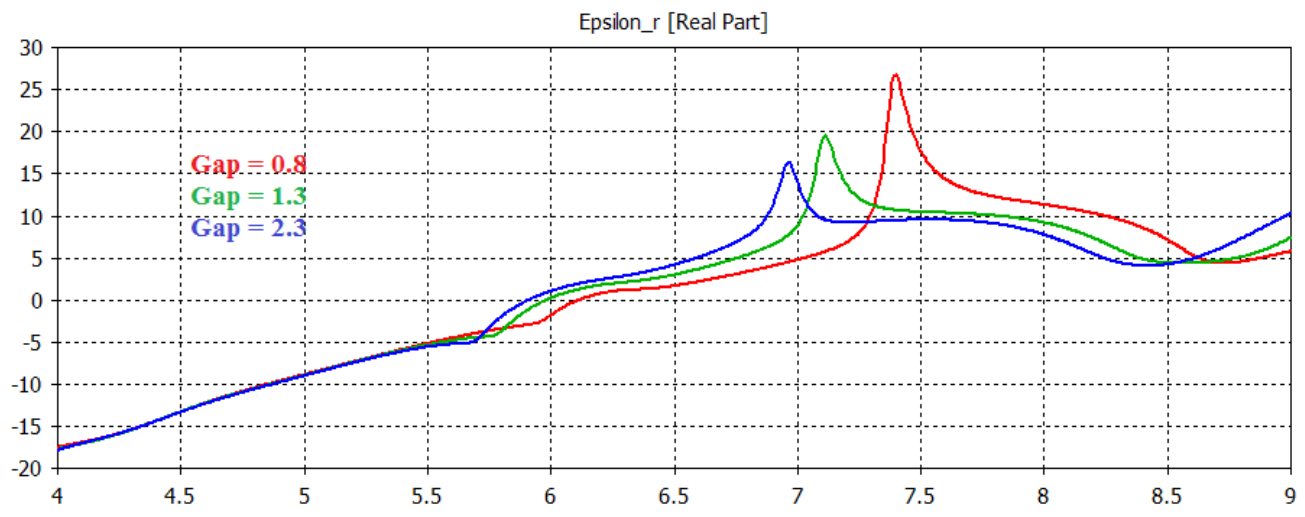

Fig 6: permeability parameter with different gap sizes

\section{Final Design and Results}

The effect of using different numbers of the metamaterial unit cells in the main beam rotation is available in Table 3.

Table 3. The effect of different numbers of unit cells

\begin{tabular}{|c|c|c|}
\hline Different Designs & $\begin{array}{c}\text { Number Unit Cell } \\
(\text { Row } \times \text { Column })\end{array}$ & Beam Rotation \\
\hline 1 & $1 \times 5$ & $3^{\circ}$ \\
\hline 2 & $2 \times 5$ & $5^{\circ}$ \\
\hline 3 & $3 \times 5$ & $7^{\circ}$ \\
\hline 4 & $2 \times 3$ & $4^{\circ}$ \\
\hline 5 & $3 \times 3$ & $6^{\circ}$ \\
\hline 6 & $3 \times 10$ & $4^{\circ}$ \\
\hline Final Design & $6 \times 5$ & $9^{\circ}$ \\
\hline
\end{tabular}

The final design is obtained by placing six rows of five of the MTM unit cells at $3 \mathrm{~mm}$ of the antenna shown in Fig 7. The results show that the best location for the metamaterial structure is $3 \mathrm{~mm}$ from the antenna to achieve a change within the main beam angle. The distance between the rows is equal to $2 \mathrm{~mm}$. 


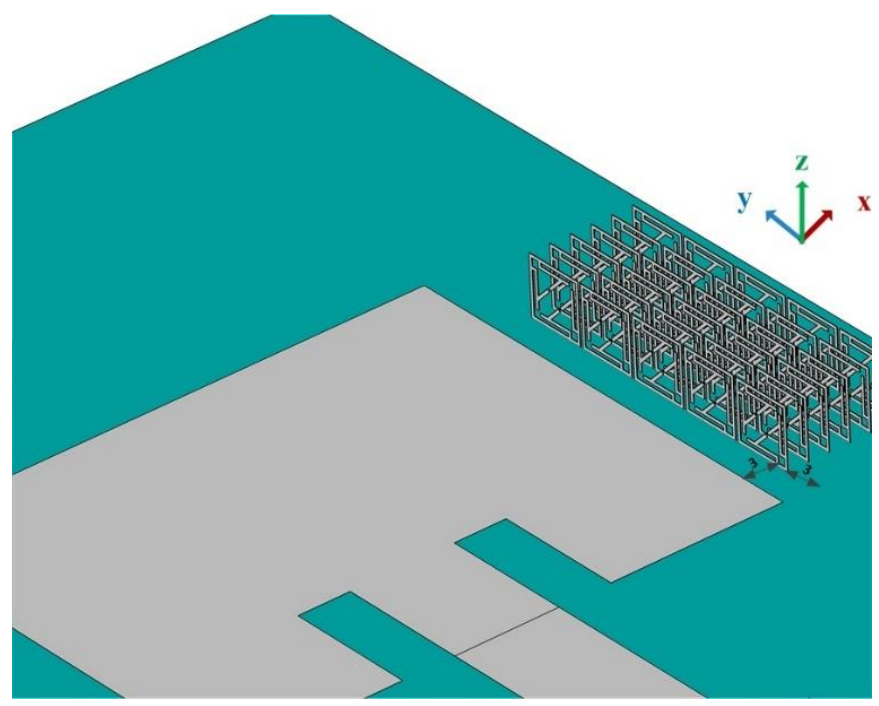

Fig. 7. Arrangement of wearable antenna.

The radiation beam of the suggested antenna loaded by MTM construction is slanted by $9^{\circ}$ in the E-plane, according to the simulation findings. Furthermore, the directivity is improved by 1.56. It is obvious from the results the antenna proposed is a suitable candidate for wearable application. In cases where the patient is in a fixed place, the direction of the beam can be controlled using the proposed wearable antenna. According to [3], SAR parameter should be lower than 1.6 for $1 \mathrm{~g}$ of tissues. The SAR value of the proposed structure is equal to 0.17 which is acceptable based on IEEE standards. The beam steering of the suggested antenna is presented in Fig. 8.

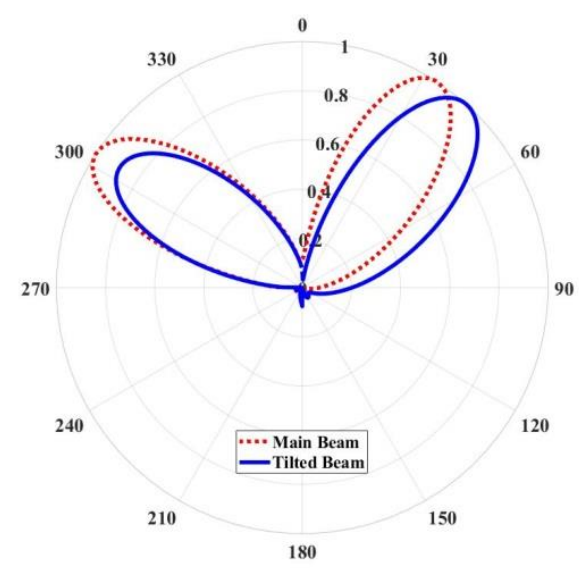

(a) 


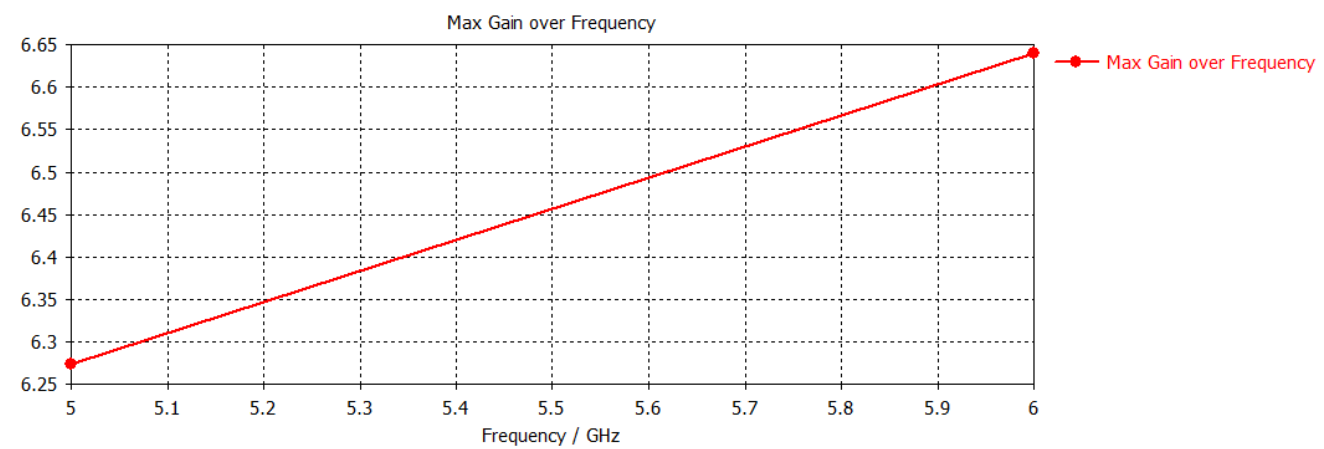

(b)

Fig 8. (a) Radiation pattern and tilted radiation pattern (b) simulation performances of the proposed antenna.

\section{Conclusion}

A new metamaterial assembly next to a wearable antenna is presented for medical applications at $5.2 \mathrm{GHz}$. This flexible antenna is located on a denim fabric as a dielectric substrate which is lightweight, widely available, and low cast. According to the wearable antennas that have been provided so far, this antenna can change the beam angle. This feature makes it possible to reduce unwanted interfaces and increase the security of the medical/telemedicine network. It also provides better network access for patients who are in a fixed place by adjusting the beam angle. This proposed structure steers the beam of the offered antenna at the angle of $9^{\circ}$ in $E$-plane. Also, the directivity is enhanced by $1.56 \mathrm{~dB}$. The simulated results of the suggested structure create it a worthy selection for medical applications.

\section{References}

[1] Z. Hamouda, J. L. Wojkiewicz, A. A. Pud, L. Koné, S. Bergheul, and T. Lasri. Magnetodielectric nanocomposite polymer-based dual-band flexible antenna for wearable applications. IEEE Transactions on Antennas and Propagation, 2018; 66(7): 3271-3277.

[2] R. B. V. B. Simorangkir, A. Kiourti, and K. P. Esselle. UWB wearable antenna with a full ground plane based on PDMS-embedded conductive fabric. IEEE Antennas and Wireless Propagation Letters. 2018; 17(3): 493-496.

[3] F. Faisal, M. Zada, A. Ejaz, Y. Amin, S. Ullah, and H. Yoo. A miniaturized dual-band implantable antenna system for medical applications. IEEE Transactions on Antennas and Propagation. 2020; 68(2): 1161- 1165.

[4] F. Khajeh-Khalili, A. Shahriari, and F. Haghshenas. A simple method to simultaneously increase the gain and bandwidth of wearable antennas for application in medical/communications systems. International Journal of Microwave and Wireless Technologies. 2020; 3(4): 374-380.

[5] P. Shirvani, F. Khajeh-Khalili, and Mohammad H. Neshati. Design investigation of a dual-band wearable antenna for tele-monitoring applications. AEU-International Journal of Electronics and Communications, 2021; 138. 
[6] B. A. F. Esmail, H. A. Majid, S. H. Dahlan, and Z. Z. abiding. Reconfigurable metamaterial structure for $5 \mathrm{G}$ beam tilting antenna applications. Waves in Random and Complex Media, latest articles. 2020; 1-14.

[7] Elfergani, I.T.E.; Hussaini, A.S.; Rodriguez, J.; Abd-Alhameed, R. Antenna Fundamentals for Legacy Mobile Applications and Beyond; Springer: Cham, Switzerland, 2017; pp. 1-659.

[8] M. Mantash, A. Kesavan, and T. A. Denidni. Millimetre-wave antenna with tilted beam for future base station applications. IET Microwave, Antennas \& Propagation. 2019; 13(2): 223230.

[9] M. K. Abdulhameed, Z. Zakaria, I. M. Ibrahim, and et al. Radiation control of microstrip patch antenna by using electromagnetic band gap. AEU-International Journal of Electronics and Communications. 2019; 110: 1-11.

[10] M. B. Kakhki, M. Mantash, A. Kesavan, M. M. Tahseen, and et al. Millimeter-wave beam-tilting Vivaldi antenna with gain enhancement using multilayer FSS. IEEE Antennas and Wireless Propagation Letters. 2018; 17(12): 2279-2283.

[11] H. Jiang, L. M. Si, and W. Hu. A symmetrical dual-beam Bowtie antenna with gain enhancement using metamaterial for 5G MIMO applications. IEEE Photonics Journal. 2019; 11(1): 1-9.

[12] B. A. Esmail, H. B. Majid, and S. H. Dahlan. Planar antenna beam deflection using low-loss metamaterial for future $5 \mathrm{G}$ applications. International Journal of RF and Microwave ComputerAided Engineering. 2019; 29(10).

[13] B. Esmail, H. A. Majid, Z. Z. abiding, and S. H. Dahlan. New metamaterial structure with reconfigurable refractive index at $5 \mathrm{G}$ candidate band. JOAM - Journal of Optoelectronics and Advanced Materials. 2019; 21(1): 101-107.

[14] J. Li, Q. Zeng, R. Liu, and T. A. Denidni. Beam-tilting antenna with negative refractive index metamaterial loading. IEEE Antennas and Wireless Propagation Letters. 2017; 16: 2030-2033.

[15] F. Khajeh-Khalili, M. A. Honarvar, M. Naser-Moghadasi, and M. Dolatshahi. Gain enhancement and mutual coupling reduction of multiple-input multiple-output antenna for millimeter-wave applications using two types of novel metamaterial structures. International Journal of RF and Microwave Computer-Aided Engineering. 2020; 30: 1-9. 